\title{
Diagnosis and Prevention of Lymphocyst
}

Lingling Liu and Shouguo Huang*

Haikou People’s Hospital, China

*Corresponding author: Shouguo Huang, professor, 13698983596,shouguohuang@126.com

\begin{abstract}
Lymphocyst is one of the most common complications following pelvic lymphadenectomy in gynecological malignancies. Lymphocyst is mostly asymptomatic. But when it grows large, the pressure symptoms such as urinary tract obstruction and venous thrombosis of the lower limbs will have a great effect on the quality of patients' life. Some lymphocyst may cause secondary infection which will affect the follow-up treatment of patients. After analyzing the domestic and international development of research of lymphocyst with an objective discussion, this paper try to find a good way of diagnosis and prevention to reduce the incidence of lymphocyst.
\end{abstract}

Key word: lymphocyst; radical surgery; gynecological tumors; diagnosis; prevention;

\section{Introduction}

Lymphocyst is one of the most common complications of gynecological malignant tumor after lymph node dissection. At present, the main point of view is that when cut off lymphatic cancer radical surgery, the distal lymph remained open so reflux lymph leakage, with intraoperative blood oozing, gathered together in the retroperitoneal space resulting in the formation of lymphocyst. It is generally accepted that the incidence of lymphocyst is about 4.3-48\%. ${ }^{1}$ The retrospective study of Achouri A included 88 patients of ovarian cancer, ${ }^{2}$ endometrial cancer, cervical cancer, who received lymph node dissection showed that the incidence of lymphocyst was in the range of $34.5 \%$. Among them, the incidence of endometrial carcinoma was the lowest, while the positive rate of lymph node was an independent risk factor. Lymphocysts can be divided into asymptomatic type and symptomatic type. Symptomatic type may be due to a large cyst, causing symptoms of oppression, such as ureteral obstruction, hydronephrosis, lower extremity edema, etc. Kondo E reviewed 321 patients after lymph node dissection. ${ }^{3}$ He found that in patients who suffered from larger than 50mm lymphocyst or continuous lymphocyst after 3 weeks, the risk of lower extremity edema, lymphangitis, and deep venous thrombosis of lower extremities were significantly increased. Therefore, the diagnosis, prevention and treatment of lymphocyst is of great significance for patients with malignant tumor radical operation.

\section{Diagnosis and differential diagnosis of lymphocyst}

It is very important to emphasize the differential diagnosis. If the recurrence or metastasis of the pelvic cavity is misdiagnosed as a cyst, the disease may be delayed. The lymphocyst was most formed in 5-8 days, no more than 23 days after the lymph node dissection. By Abdominal palpation, bimanual and unilateral or bilateral abdominal cystic mass in the inguinal area was round or oval, tension. The lymphocyst should be distinguished from tumor, cystadenoma, adnexal cyst, pelvic abscess, pelvic thrombophlebitis and cystic metastasis phase identification. Under the guidance of B ultrasound, puncturing cyst and draining lymph can be used as a diagnostic method for the diagnosis of lymphocyst. However, there are 
contraindications to puncture, such as the recurrence of ovarian cancer should not accept such treatment. Therefore, B ultrasound and MRI can be used as the main means of auxiliary diagnosis. The following main characteristics of Ma Lili summarized ultrasound lymphocyst: ${ }^{4}$ 1 , the shape is round, oval, regular; 2 , the cyst wall is thin and the wall is not smooth; 3 , sac sound better, part of the visible band separated; 4, no blood flow signals were detected in cystic wall; 5 . Located in the bilateral iliac fossa, anterior medial and lateral iliac artery. The dissections of 69 patients suffered from lymphocyst were examined by MRI. Niu Lei draw the conclusions: ${ }^{5} 1$, plain scan showed round or oval long T1 and long T2 signal; 2, the characteristics of DWI high signal diffusion restriction site 3, cyst distributed Along the wall of the basin distribution, and accompanied the external iliac arteries and veins. It can be seen that the B ultrasound and MRI manifestations of lymphocysts are special and can be used as the basis for diagnosis and differential diagnosis.

\section{The prevention of the formation of lymphocyst}

The most common prevention methods from the causes of the formation of lymphatic cyst are to reduce the production of lymph and increase its absorption. The discussion focuses on the operation of closed lymphatic vessels, retroperitoneal treatment, retroperitoneal drainage tube, the use of biological protein glue, etc.Some of them have been used in clinic as usual, and some of them still need to be verified by a larger number of experiments.

\subsection{Closed lymphatic vessels}

The cause of the formation of lymphocyst for pelvic lymph node dissection, the small lymphatic vessels open, reflux lymph drainage is not good. Closed lymphatic vessels play a role in reducing lymph leakage. The main methods of ligation, avulsion, electrocoagulation, ultrasonic vaporization method, BiClamp method. It is generally believed that the ligation of lymphatic vessels can block the lymphatic drainage of the lower limbs, reduce the accumulation of lymph, and reduce the incidence of lymphocyst. Lu Huaiwu randomly selected 32 patients with standardized gynecological malignant tumor and random ligaturedleft or right deep inguinal lymphatic, ${ }^{6}$ proximal obturator lymph vessels, obturator lymph vessels, distal iliac lymphatic, internal and external iliac vein at the intersection of lymphatic vessels. After 1, 4, 12 and 24 weeks respectively, the situation of the open and the ligated lateral lymphocysts was examined by pelvic ultrasound .This self-controlled study excluded patients with personal factors, the situation of operation, the anatomic structure on both sides of the pelvic and other factors. The conclusions are as follows: ligation of pelvic lymph node in the near future can reduce the incidence of lymphocyst. Feng Yan furtherly simplified the 5 ligation sites of 5 pelvic lymph nodes to 2 sites. $^{7}$ The 2 sites were the proximal and distal sites of obturator lymph nodes. Patients were randomly divided into the ligation group and part ligation group. Part ligation group had a significantly higher incidence of the lymphocyst than ligation group. Conclusion: the method of ligaturing 5 lymph sites of iliac lymphatic trunk is effective. Compared with the two groups of patients with lymph node dissection by ligation and avulsion, the incidence of the lymphocyst was significantly lower in the ligation group. The difference had statistical significance. Simforoosh N etal taken 13 patients with lymph node dissection as the research object. ${ }^{8}$ Then found that tusing bipolar coagulation as lymph node dissection means does not increase the formation of lymphatic leakage and lymphocyst conclusion. But because of the small number of cases, it still needs 
further study with larger sample. As a new type of instrument, the advantage of the harmonic scalpel is that it can make the tissue water vaporize, the cell is decomposed, and the tissue can be separated and cut more accurately. Rafii A etal Selected 98 patients who underwent laparoscopic extraperitoneal lymphadenectomy, ${ }^{9}$ including 62 cases of A group using conventional methods of lymph node dissection, 36 cases of B group by harmonic scalpel lymph node dissection. In A group, there were 7 patients who had symptomatic lymphocyst while in B group there were no such patients statistically. The difference had statistical significance. The results of this study show that pelvic lymph node dissection with a harmonic scalpel is helpful to reduce the incidence of postoperative symptomatic lymphocyst. Thus, it is necessary to close the lymphatic vessels. The method of ligation and ultrasonic vaporization has more advantages in reducing the incidence of lymphocyst.

\subsection{The opening of the retroperitoneum}

$\mathrm{Li}$ Yan and so on studying various of factors of lymphocyst by regression analysis, ${ }^{10}$ found that the treatment of retroperitoneal is an independent factor affecting the incidence of lymphocyst. At present, there is no unified opinion. The traditional view of the retroperitoneal suture can reduce the risk of infection, pelvic adhesions and other risks. More and more scholars believe that peritoneal cavity may be induced by suturing the peritoneum. And suturing too tight may cause ischemia of surrounding tissue, fluid accumulation, and poor drainage, more likely to cause lymphocyst. A research was setted up two groups: A and B. In A group they placed the vaginal drainage tube after the closure of peritoneum, while in $\mathrm{B}$ group they kept the posterior peritoneum open without the vaginal drainage. Results: After the closure of the peritoneal the incidence of lymphocyst had increased, but the incidence of symptomatic Lymphocyst did not increase. The closure of peritoneum reduced the possibility of the formation of pelvic infection and ascites. Wu Yanli et al. study showed that keep the posterior peritoneum open can shorten the operation time and reduce the incidence of Lymphocyst. At present, the treatment of the posterior peritoneum needs larger samples to be studied, and a unified opinion should be further formed.

\subsection{Retroperitoneal drainage tube}

Although there is no clear evidence, the traditional view is that the placement of retroperitoneal drainage tube can reduce the incidence of lymphocyst. With the deepening of the research, domestic and foreign researchers have conducted a number of experiments. Charoenkwan $\mathrm{K}$ taken a retrospective study of patients underwented gynecological pelvic lymph node dissection before May 2009 showed that: ${ }^{11,12} 4$ weeks after the operation, when the pelvic peritoneum remains open, the rates of formation of lymphocyst and symptomatic Lymphocyst were higher in the patients who were placed the vaginal drainage tube than those in the other patients without the vaginal drainage tube. 12 months after operation, the rates of formation of lymphocyst in the two groups were the same, while the rate of formation of symptomatic Lymphocyst was higher in the group without the vaginal drainage tube. This study concluded that: when keeping the retroperitoneum open, the placement of retroperitoneal drainage tube will increase the rate of recent formation of the lymphocyst, and the incidence of symptomatic Lymphocyst. More and more scholars suggest that retroperitoneal drainage tube should not be placed at the same time. 


\subsection{The application of fibrin glue}

Fibrin glue or fibrin sealant can form biological membrane in the wound. When it was to be used in dissection, it could isolate wound from normal tissues, reduced lymph leakage to reduce the incidence of lymphocyst. However, Scholz et al. took a target study by dividing 93 patients into two groups. They found that the use of fibrin glue in the formation of lymphatic cyst and subsequent complications was not effective. ${ }^{12}$ Therefore, the effect of fibrin glue still needs more experiments to further verify.

\subsection{Omentoplasty}

Because of the good blood circulation of omentum majus, strong regeneration ability, absorbing ability, researchers pulled omentum into the pelvic cavity, placed it between the bowel and bladder, to absorb the leakage of lymph and reduce the formation of lymphocyst. Saad et al. selected 4 patients with persistent lower limb lymphedema and invalid conservative treatment to perform omentoplasty. ${ }^{13}$ The lymphedema of the 4 patients were improved significantly after operation.

\subsection{Detection of sentinel lymph node (SLN)}

Sentinel lymph node refers to the first site of tumor drainage and tumor metastasis. At home and abroad, the application of sentinel lymph node in gynecological tumors has been studied in many aspects. Radical resection of a malignant tumor may increase the incidence of complications such as lymphocyst. The earliest use of sentinel lymph node detection was in vulvar cancer. It is associated with the incidence of postoperative complications of vulvar cancer, sentinel lymph node detection rate and transfer characteristics. Van der Zee observed 403 patients with vulvar cancer by multicenter observation. It was found that the incidence of lymphocyst was $1.9 \%$ in patients with only SLN, and the incidence of patients with inguinal lymph node was as high as $25.2 \%$.Lymphatic metastasis is an important way of cervical cancer metastasis. There are many researches on the application of sentinel lymph node mapping in the study of cervical cancer patients to confirm the clinical feasibility and reliability. The study found that the negative predictive value of sentinel lymph node biopsy in the patients with cervical cancer whose tumor diameter less than $20 \mathrm{~mm}$, was close to $100 \%$. So for this in a kind of patients, sentinel lymph node technology is a reliable method to judge whether lymph node involvement. Sentinel lymph node mapping techniques have significant advantages in reducing surgical complications.

\section{Conclusions}

In the prevention and treatment of lymphocyst, it is the fundamental solution to prevent lymphocyst from the cause of formation.The discussion focuses on the operation of closed lymphatic vessels, retroperitoneal treatment, retroperitoneal drainage tube, the use of biological protein glue, etc. But the prevention of lymphocyst is still a difficult problem in gynecologic oncology.We still need a larger sample of domestic and international experts to find the best way to prevent lymphocyst.

\section{References:}

1. Y. Tsuji, R. Kawaguchi, S. Haruta, et al. Late onset of pulmonary embolism caused by 
lymphocyst following pelvic lymphadenectomy, Journal of Obstetrics \& Gynaecology Research, 33(2007):734-738.

2. A. Achouri, C. Huchon,Complications of lymphadenectomy for gynecologic cancer, European Journal of Surgical Oncology the Journal of the European Society of Surgical Oncology \& the British Association of Surgical Oncology, 39(2013):81-6.

3. E. Kondo, T. Tabata, T.Shiozaki, et al., Large or persistent lymphocyst increases the risk of lymphedema, lymphangitis, and deep vein thrombosis after retroperitoneal lymphadenectomy for gynecologic malignancy, Archives of Gynecology and Obstetrics, 288(2013):587-93.

4. M. Lili, The value of ultrasonography in the diagnosis of lymphocyst after dissection in gynecologic malignancies,Clinical Medicine, 32(2012):109-110.

5. L. Niu, e tal. , MRI diagnosis of pelvic lymphocyst after pelvic lymph node dissection, Pract Radiol, 28(2012):914-915.

6. H.W. Lu, et al., Influence of Formation of Lymphcyst Following Ligation of Lymphatic vessels in Pelvic Lymph Nodes Dissection,JOURNAL OF SUN YAT-SEN UNIVERSITY（MEDICAL SCIENCES）,30(2009): 758-761.

7. Y.Feng, The incidence of lymphocyst following systematiclymphadenectomy with drainage, with ligation of lymph vessels, Sun Yat-Sen University, 2009.

8. N.Simforoosh, H. Nasseh, P. Masoudi, et al., Clipless laparoscopic retroperitoneal lymph node dissection using bipolar electrocoagulation for sealing lymphatic vessels: initial series, Urology Journal, 9(2012):480-5.

9. A.Rafii, A. Camicas, G. Ferron, et al., A comparative study of laparoscopic extraperitoneal laparoscopy with the use of ultrasonically activated shears, American Journal of Obstetrics \& Gynecology, 201(2010):370.e1-5.

10. Y.Li,P.Huang,et al., Comparision of Diffenent Surgical Management of Retroperitoneum after Systemic Pelvic Lymphadenectomy, Chinese Journal of Clinical Oncology,36(2009):887-890.

11. K.Charoenkwan, C. Kietpeerakool, Retroperitoneal drainage versus no drainage after pelvic lymphadenectomy for the prevention of lymphocyst formation in patients with gynaecological malignancies, Cochrane Database of Systematic Reviews, 6(2014):CD007387.

12.H.S. Scholz , E.Petru , C.Benedicic,J. Haas ,K. Tamussino ,R. Winter, Fibrin application for preventing lymphocysts after retroperitoneal lymphadenectomy in patients with gynecologic malignancies. Gynecol Oncol. 84(2002):433-6.

13. S. M. Attash, M. Y. Alsheikh, Case Report: Omental flap for treatment of long standing lymphoedema of the lower limb: can it end the suffering? Report of four cases with review of literatures, Case Reports, 2012, 2013(feb08 1). 